\title{
Radial extracorporeal shockwave therapy for the treatment of finger tenosynovitis (trigger digit)
}

This article was published in the following Dove Press journal:

Open Access Journal of Sports Medicine

31 October 2016

Number of times this article has been viewed

\author{
Nikos Malliaropoulos ${ }^{1-5}$ \\ Rosanna Jury ${ }^{1,2,5}$ \\ Debasish Pyne ${ }^{3-5}$ \\ Nat Padhiar ${ }^{3,5}$ \\ Jennifer Turner ${ }^{6}$ \\ Vasileios Korakakis ${ }^{3,7}$ \\ Maria Meke' \\ Heinz Lohrer ${ }^{3,8}$ \\ 'Sports and Exercise Medicine, \\ Thessaloniki Musculoskeletal Clinic, \\ ${ }^{2}$ Thessaloniki National Track and \\ Field Centre, Sports Medicine Clinic \\ of S.E.G.A.S., Thessaloniki, Greece; \\ ${ }^{3}$ European Sports Care, ${ }^{4}$ Department \\ of Rheumatology, Sports Clinic, \\ Barts Health NHS Trust, ${ }^{5}$ Centre \\ for Sports \& Exercise Medicine, \\ Queen Mary University of London, \\ London, UK; ${ }^{6}$ Healthcare Group \\ Guernsey, Guernsey; ${ }^{7}$ Aspetar, \\ Orthopaedic and Sports Medicine \\ Hospital, Doha, Qatar; ${ }^{8}$ European \\ SportsCare Network (ESN), Zentrum \\ für Sportorthopädie, Wiesbaden- \\ Nordenstadt, Germany
}

Correspondence: Rosanna Jury Centre for Sports \& Exercise Medicine, Queen Mary University of London, Bancroft Road, London EI 4DG, UK Tel +447969070470

Email rj6g10@soton.ac.uk
Introduction: Stenosing tenosynovitis that is characterized by the inability to flex the digit smoothly, usually leads to prolonged rehabilitation or surgery.

Study design: This case series is a retrospective cohort study.

Purpose: The aim of this case series was to evaluate the effectiveness of radial extracorporeal shockwave therapy (rESWT) for the treatment of stenosing tenosynovitis of the digital flexor tendon (trigger digit).

Methods: A retrospective analysis of 44 patients (49 fingers) treated with an individually adapted rESWT protocol was conducted. Trigger digit pain and function were evaluated at baseline and 1-, 3-, and 12-months posttreatment. Recurrence and pretreatment symptom duration were analyzed. Results: Significant reductions in pain scores and functional improvement were found between baseline and all follow-up assessments $(P<0.001)$. Pretreatment symptom duration was significantly correlated with the number of rESWT sessions required $(r=0.776, P<0.001)$ and 1 -year posttreatment pain score $(r=0.335, P=0.019)$.

Conclusion: This study provides initial evidence that rESWT is an effective treatment for trigger digit, but randomised controlled trials are required to provide further evidence of this effect. Keywords: trigger finger, trigger thumb, tendon, shockwave therapy, non-surgical therapy, conservative treatment, physiotherapy

\section{Introduction}

Trigger digit, also known as stenosing tenosynovitis, is characterized by an inability to flex or extend the digit smoothly, often with pain over the palmar aspect of the metacarpophalangeal joint. ${ }^{1-3}$ This condition develops when thickening of the tendon sheath at the first annular ligament's pulley and subsequent constriction of the tendon prevents it from gliding through. Pinching of the tendon often causes nodule formation, ${ }^{1,3,4}$ and patients typically present with a locking, popping sensation as the nodule catches at the constriction. ${ }^{1,2,4}$ In some cases, it resolves spontaneously; however, if left untreated, trigger digit may gradually progress until the affected finger or thumb is permanently locked in flexion. ${ }^{1}$ Histologically, the A1 pulley exhibits fibrocartilaginous metaplasia, and in the tendon tissue, areas of hyalinosis, mucoid degeneration, and chondral metaplasia are found.$^{5}$ It is the most common flexor tendinopathy, ${ }^{2}$ with highest incidence in women (75\%) and in those aged between 52 and 62 years, ${ }^{6}$ with the fourth digit (ring finger) and thumb most often affected. ${ }^{3}$

Trigger digits are usually idiopathic; however, some authors argue that there is a possible correlation with excessive use of the hand. ${ }^{7,8}$ Other potential risk factors 
include rheumatoid arthritis, diabetes mellitus, carpal tunnel syndrome, Dupuytren's disease, amyloidosis, hypothyroidism, mucopolysaccharide storage disorders, congestive heart failure, and genetic predisposition. ${ }^{4,9,10}$

Treatment aims to eliminate pain and stop triggering. Currently accepted conservative treatments include pain medication, orthoses, physiotherapy, and corticosteroid injections. ${ }^{3}$ Corticosteroid injection has the greatest success rate; ${ }^{11}$ however, it is still reported to be effective only in $57 \%$ of the patients. ${ }^{6}$ Moreover, multiple injections may cause tendon rupture, a course of more than two injections is reported to almost never be successful, and repeated injections before surgery are not cost-effective. Thus, for many patients, corticosteroid injection is not a long-term treatment option. ${ }^{2,12,13}$

Surgical treatment involves percutaneous and open release of the A1 pulley. ${ }^{2,3}$ Both are reported to be similarly effective and superior to corticosteroid injection, ${ }^{14}$ with remission of symptoms achieved in $97 \%-100 \%$ of cases. ${ }^{14-17}$ However, it is associated with longer recovery times and more complications including tendon bowstringing, digital ulnar drift, and nerve injuries. ${ }^{3}$ Due to these possible complications and as guidelines by the American College of Occupational and Environmental Medicine ${ }^{18}$ recommend surgery only when conservative treatments have failed, radial extracorporeal shock wave therapy (rESWT) could play a role as a noninvasive alternative.

rESWT is a noninvasive and safe therapy and has been used in the management of tendon pathologies since the early $1990 \mathrm{~s}$ in order to alleviate pain and improve function. A radial shockwave is a low-energy wave that is generated when a projectile in the hand piece of the rESWT device is accelerated by a compressed air source and hits a metal applicator. The pressure wave is transmitted to the patient's skin through ultrasound gel and spreads as a spherical (radial) wave into the tissue. The energy is highest at the tip of the applicator and decreases peripherally by the square of the distance. ${ }^{19-21}$ The biological mechanisms by which rESWT induces therapeutic effects on pathological tendon tissue are not completely elucidated, although it is suggested that shockwaves may facilitate the healing process through the disruption of avascular, damaged tissues, stimulation of growth factor release, stem cell recruitment, and neovascularisation. ${ }^{22-25}$

rESWT has been used to treat numerous other tendinopathies, such as epicondylitis, ${ }^{22,26}$ plantar fasciitis, ${ }^{26-28}$ patellar tendinopathy, ${ }^{21,26,29}$ shoulder calcific tendinitis, ${ }^{19,30,31}$ proximal hamstring tendinopathy, ${ }^{32}$ and medial tibial stress syndrome. ${ }^{20,33}$ However, to the authors' knowledge, to date, no studies on rESWT and trigger digit have been conducted.
Acknowledging the poor efficacy and the prolonged rehabilitation of traditional conservative treatments, possible complications associated with surgery, and the evidence that rESWT (with the additional advantage that it is noninvasive) is effective in the treatment of other tendinopathies, the aim of this study was to determine whether rESWT is a safe and effective treatment for trigger digit. Therefore, the functional improvement and mean pain reduction over a 1-year followup period were assessed. In addition, the individualised treatment protocol was retrospectively analysed to look for correlations between pre-treatment symptom duration, the number of rESWT sessions required until recovery, and the 1 -year post-treatment visual analog scale score.

\section{Materials and methods}

\section{Study subjects}

Ethical approval for this retrospective, uncontrolled cohort study was granted by the Thessaloniki Clinic Institutional Review Board (ART No 2012-03). All participants provided written informed consent. The study included 44 adults (49 fingers/thumbs; 39 adults with single-digit pathology and five with two-digit pathology) who were sequentially diagnosed with trigger digit by a consultant in Sport and Exercise Medicine outpatient clinic between 2009 and 2013 (Table 1). The diagnosis was based on clinical history and examination. Patients underwent ultrasound imaging to assess thickening of the A1 pulley.

The mean age of the study participants was $49 \pm 10$ years (ranging from 32 to 71 years). Of the total participants, 33 par-

Table I Characteristics of patients and trigger digits receiving radial extracorporeal shockwave therapy

\begin{tabular}{ll}
\hline Characteristics & \\
\hline Sex & Patients (44) \\
Male (\%) & $15(34.1 \%)$ \\
Female (\%) & $29(65.9 \%)$ \\
Hand affected: & Patients $(44)$ \\
Right (\%) & $26(59.1 \%)$ \\
Left $(\%)$ & $13(29.5 \%)$ \\
Bilateral $(\%)$ & $5(11.4 \%)$ \\
Digit affected & Fingers/thumbs $(49)$ \\
Thumb & $22(44.9 \%)$ \\
Index & $5(10.2 \%)$ \\
Third finger & $7(14.3 \%)$ \\
Fourth finger & $13(26.5 \%)$ \\
Fifth finger & $2(4.1 \%)$ \\
Quinnell's grade & Fingers/thumbs $(49)$ \\
Grade 2 (actively correctable triggering) & $24(48 \%)$ \\
Grade 3 (passively correctable triggering) & $25(52 \%)$ \\
Age (years), mean ( \pm SD) & $49.06( \pm 9.99)$ \\
Duration of pain (months), mean $( \pm S D)$ & $5.09( \pm 5.72)$ \\
\hline
\end{tabular}

Note: Values are counts, number of patients (percentages) unless stated otherwise. 
ticipants (67.3\%) were women and 16(32.7\%) were men. The average pretreatment symptom duration was $5 \pm 5.7$ months (ranging from 1 to 24 months); 19 fingers/thumbs (38.8\%) had symptoms for $<3$ months, 19 fingers/thumbs (38.8\%) from 3 to 6 months, 4 fingers/thumbs $(8.2 \%)$ from 6 to 12 months, and $7(14.3 \%)$ fingers/thumbs from 12 to 24 months.

\section{Inclusion criteria}

Patients with Grade 2 and Grade 3 trigger digit according to Quinnell's classification (Grade 0: normal finger movements, Grade 1: uneven finger movements, Grade 2: actively correctable triggering, Grade 3: passively correctable triggering, and Grade 4: locked digit) were included. ${ }^{34}$

\section{Exclusion criteria}

Patients with Quinnell's Grade 1 and Grade 4 trigger digit were excluded from the study, and Grade 4 was referred for surgery. The exclusion criteria were as follows: traumatic trigger digit, age $<18$ years, rheumatoid arthritis, generalized polyarthritis, local infection, and history of malignancy, cervical pain, or previous surgery for trigger digit. Possible differential diagnoses such as infective tenosynovitis, metacarpophalangeal articular pathology, gamekeeper's thumb, and Dupuytren's contracture were excluded by clinical examination and ultrasound imaging.

\section{Procedure and equipment}

Treatment was administered by using the Storz Medical Masterplus ${ }^{\circledR}$ MP200 (Storz Medical, Tägerwilen, Switzerland) rESWT device. At each session, 2,000 impulses were applied to the painful area overlying the pathological flexor tendon at a frequency of $5-6 \mathrm{~Hz}$. The device pressure varied from 1 to
3 bars depending on the patient's individual pain tolerance; it was kept low enough to ensure that pain was tolerable, but high enough to have a therapeutic effect. Treatment sessions took place weekly until symptoms subsided, and thus, the total number of treatment sessions for each patient were based on the individual response to treatment.

Ultrasound gel was used for the transmission of shockwaves to the patient's skin. No local anesthesia was administered as it is known to reduce the efficacy of ESWT. ${ }^{27,28,35,36}$ The painful area was covered in a longitudinal pattern starting at the point of maximum pain determined by patient's feedback. At the end of each session, the patients were advised to use the finger normally, and they underwent no other treatments during the follow-up period of the study. All the patients were free to seek additional care (surgical consultation) if unsatisfied with improvement.

\section{Outcome measures and follow-up}

Patients were assessed at baseline (pretreatment) and at 1-, 3-, and 12-months follow-up appointments (posttreatment) when a full clinical examination was performed (Figure 1). All patients were present during the follow-up period. The Roles and Maudsley score was used to assess functional outcome after rESWT treatment. This is a 4-point scale where 1 indicates an "excellent" result, 2 a "good" result, 3 a "fair" result, and 4 a "poor" posttreatment result. "Excellent" and "good" results were considered a success, meaning that the patients had full movement of the affected finger and activity was not restricted. "Fair" and "poor" results were considered a failure, bearing identical or even worse symptoms after rESWT treatment. These cases experienced some discomfort after prolonged activity or pain-limited activities. ${ }^{37}$

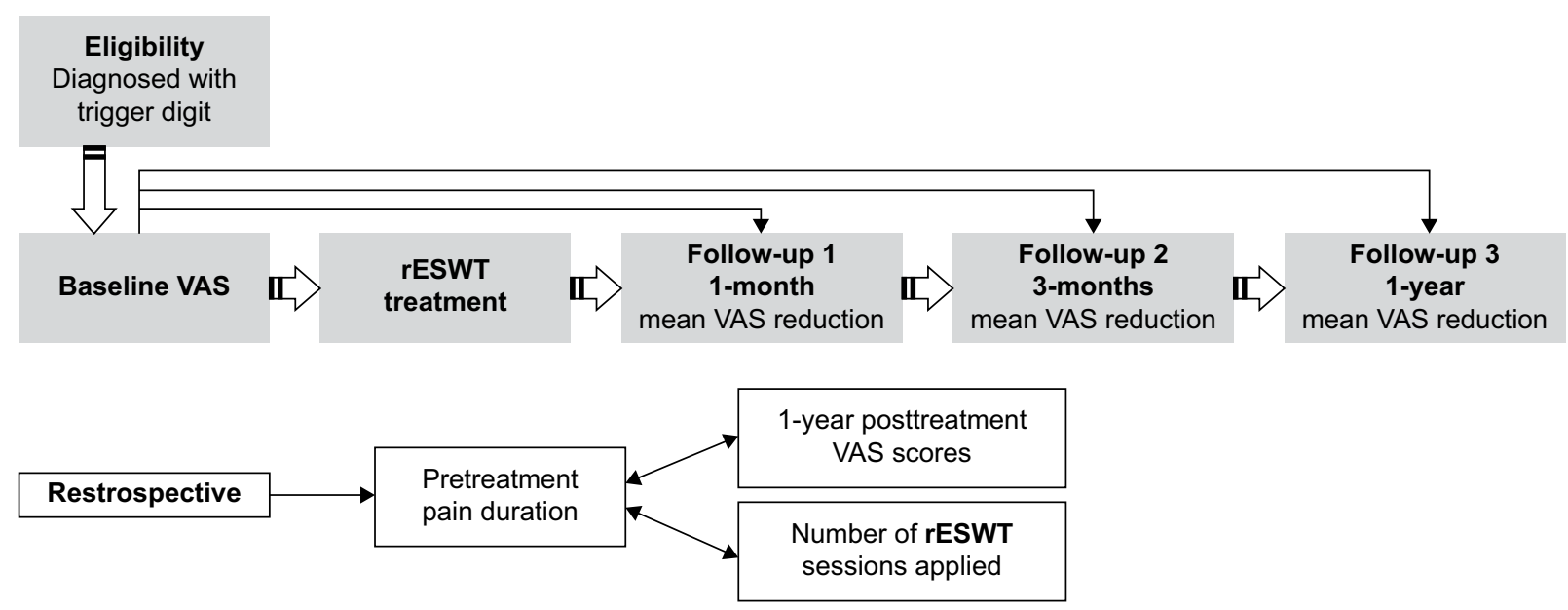

Figure I Research study design and stages.

Notes: Unidirectional arrows indicate the sequential stages of the study. Bidirectional arrows indicate that correlation between variables was examined. Abbreviations: rESWT, radial extracorporeal shockwave therapy; VAS, visual analog scale. 
Pain severity was measured using the standard VAS. A score of 10 indicated maximum pain and 0 the minimum. Primary outcome measures were functional improvement and mean VAS reductions at 1-, 3-, and 12-months posttreatment compared with baseline. A VAS reduction of $60 \%$ was considered a clinically positive result. Recurrence of symptoms was defined as a 1-year follow-up VAS score of $\geq 2$.

The individualized protocols were also retrospectively analyzed in terms of the number of sessions required and the pressure used (bars). To do this, additional outcome measures were used to assess correlations, first, between pretreatment symptom duration and the number of rESWT sessions required until recovery and, second, between pretreatment symptom duration and the 1-year posttreatment VAS score.

\section{Statistics}

Descriptive analysis was conducted, aiming at a comprehensive presentation of the patients' demographics (such as age and sex) and the distribution of the affected hands and fingers. Total number of rESWT sessions required, shockwave impulses, and pressure and frequency used were retrospectively analyzed as means. Mean VAS scores were calculated at each follow-up interval, and mean VAS reductions were assessed from the percentage decrease in mean pain level from the pretreatment (baseline) VAS. Significant differences between baseline VAS and follow-up VAS scores were assessed by using Wilcoxon signed rank test, and Monte Carlo simulation was used to test statistical significance. Quartile statistics were also used to describe VAS variables as a nonparametric test was used.

In order to assess the relationship between pretreatment pain duration (in months), the number of rESWT sessions required, and the 1-year follow-up VAS score posttreatment, Spearman's rank correlation coefficient was used.

A combination of Statistical Package for the Social Sciences Version 20 (IBM Corporation, Armonk, NY, USA) and Microsoft Excel (Microsoft Corporation, Redmond, WA, USA) was used to conduct all statistical analyses, and the confidence level was predefined at $95 \%(\alpha=0.05)$ for all statistical analyses. $^{38,39}$

\section{Results}

The average number of rESWT sessions required was $6 \pm 1.3$ (ranging from 3 to 8 ). All the 44 subjects (49 digits) were treated with 2,000 impulses of shockwave per session at a mean pressure of $1.4 \pm 0.3$ bar (ranging from 1 to 3 ) and a mean frequency of $5 \pm 0.4 \mathrm{~Hz}$ (ranging from 5 to 6 ; Figure 2).

From the total of 49 digits, one (2\%) received three sessions, nine (18.4\%) received four sessions, $12(24.5 \%)$ received five sessions, $20(40.8 \%)$ received six sessions, and $7(14.3 \%)$ received eight sessions.

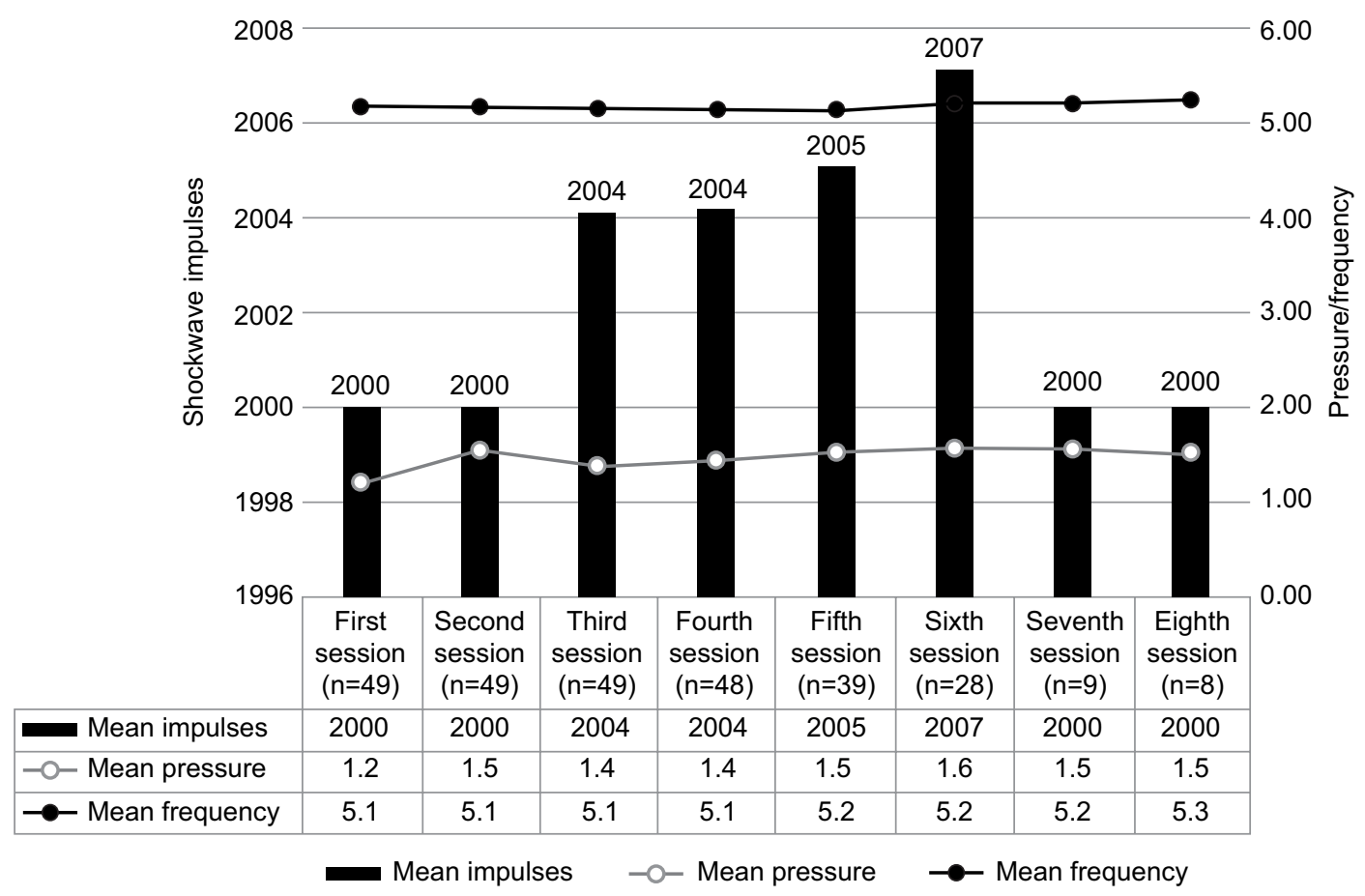

Figure 2 Radial extracorporeal shockwave therapy mean impulses, mean pressure, mean frequency per session, and number of digits (fingers or thumbs) contributing to each successive session. 
According to the Roles and Maudsley score, $73.5 \%$ (36) of the patients stated their functional post-rESWT treatment condition as "excellent" and 20.4\% (10) as "good" (Table 2). A "fair" functional post-rESWT treatment condition was stated in three cases (6.1\%).

A statistically significant reduction in VAS scores was found between baseline and 1-month follow-up $(z=-6.212$, $P<0.001)$, between baseline and 3-month follow-up $(z=-6.220, P<0.001)$, and between baseline and 1-year follow-up $(z=-6.209, P<0.001)$. All the 49 cases had negative ranks with a mean rank of 25.00 in all cases. Pain score rating was 8.0 at baseline, 3.0 at 1-month posttreatment, and 1.0 both at 3-month and 1-year follow-up (Figure 3 and Table 3).

Three patients had recurrent symptoms at 1-year followup with the recurrence rate estimated at $6.1 \% ; 34.7 \%$

Table 2 Roles and Maudsley score functional radial extracorporeal shockwave therapy treatment results

\begin{tabular}{lll}
\hline Grade & Number of cases & Percent \\
\hline Excellent & 36 & 73.5 \\
Good & 10 & 20.4 \\
Fair & 3 & 6.1 \\
Poor & 0 & 0 \\
Total & 49 & 100.0 \\
\hline
\end{tabular}

(17 digits) had a VAS score of 0 (asymptomatic), 59.2\% (29 digits) had a VAS score of 1.0, and 4.1\% (two digits) had a VAS score of 2.0. In addition, no complications were reported in the subjects.

A strong positive Spearman's $\rho$ correlation was found between pre-rESWT treatment symptoms duration and the number of sessions required $(r=0.776, P<0.001$; Figure 4$)$.

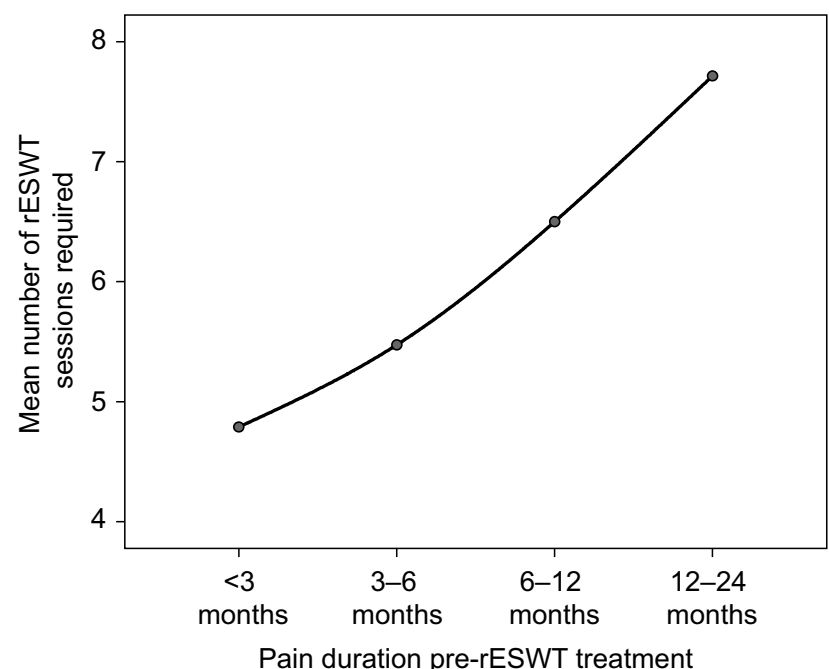

Figure 4 Pre-radial extracorporeal shockwave therapy symptoms duration versus the number of sessions required.

Abbreviation: rESWT, radial extracorporeal shockwave therapy.

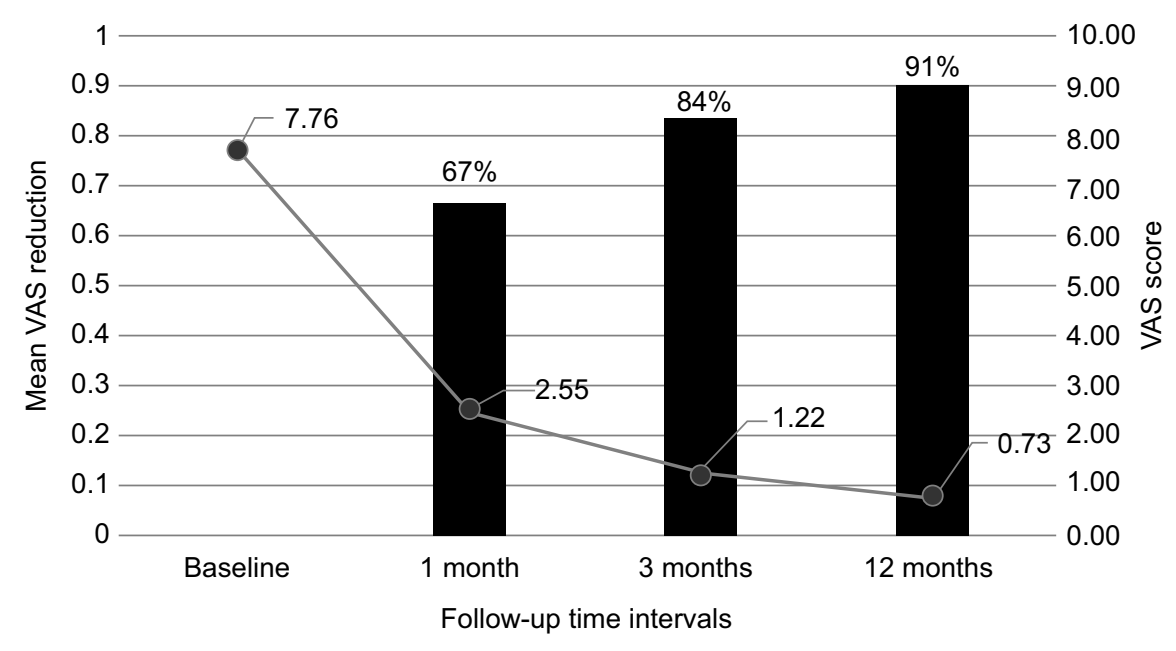

Mean VAS reduction

Average pain level (1-10 VAS score)

Figure 3 Radial extracorporeal shockwave therapy mean VAS reduction and average pain level at follow-up time intervals. Abbreviation: VAS, visual analog scale.

Table 3 Visual analog scale (VAS) scores means, standard deviation, and variance at baseline and follow-up

\begin{tabular}{|c|c|c|c|c|c|c|c|c|}
\hline & \multirow[t]{2}{*}{$\mathbf{n}$} & \multirow[t]{2}{*}{ Mean } & \multirow{2}{*}{$\begin{array}{l}\text { Standard } \\
\text { deviation }\end{array}$} & \multirow[t]{2}{*}{ Minimum } & \multirow[t]{2}{*}{ Maximum } & \multicolumn{3}{|c|}{ Percentiles } \\
\hline & & & & & & 25th & 50th (median) & 75th \\
\hline Baseline_VAS & 49 & 7.8 & 0.7 & 7 & 9 & 7.0 & 8.0 & 8.0 \\
\hline VAS_Ist month & 49 & 2.6 & 0.6 & 1 & 4 & 2.0 & 3.0 & 3.0 \\
\hline VAS_3rd month & 49 & 1.2 & 0.8 & 0 & 3 & 1.0 & 1.0 & 2.0 \\
\hline VAS_I year & 49 & 0.7 & 0.6 & 0 & 3 & 0.0 & 1.0 & 1.0 \\
\hline
\end{tabular}


Significant, positive, but weak correlation was also found to 1 -year VAS with pretreatment symptoms duration $(r=0.335$, $P=0.019$ ).

\section{Discussion}

Trigger digit caused by fibrocartilaginous metaplasia of the tendon sheath can be a chronic and progressive condition. ${ }^{1-3}$ In the literature spontaneous recovery has been reported in $20 \%-29 \%$ of cases; however, in some untreated patients, the initial symptoms of intermittent pain, snapping or triggering progress over time, and long-standing cases may present with the affected finger or thumb locked in flexion. ${ }^{1}$

According to the authors' knowledge, this is the first study conducted to assess the effectiveness of rESWT for trigger digit. Evidence from previous studies has found that rESWT is effective in the treatment of multiple other tendinopathies. ${ }^{19-33,40,41}$ However, the specific biological mechanisms by which it induces therapeutic effects on pathological tendon tissue are not completely elucidated. Evidence from a recent study of human tendons suggested that the mechanical stimulus of ESWT aids tendon remodeling by promoting inflammatory and catabolic processes that are associated with the removal of damaged matrix constituents. ${ }^{42}$ In vitro studies have further suggested that ESWT increases tendon healing through the stimulation of cell proliferation and motility, neovascularization, and expression of critical differentiation genes. ${ }^{43}$ However, these in vitro findings cannot be automatically generalized to the in vivo conditions in human tendinopathies.

In the present study, statistically significant pain reductions $(P<0.001)$ and functional improvements were found through all midpoints during the 1-year follow-up in $93.1 \%$ of cases, whereas only three patients had recurrent symptoms at 1-year posttreatment assessment. According to this initial evidence, rESWT was proven to be a suitable treatment for trigger digit. The analysis of the individualized treatment protocols demonstrated that longer pretreatment symptom duration was positively correlated with more sessions required until recovery $(P<0.001)$ and a higher VAS score at 1 -year posttreatment $(P<0.05)$.

\section{rESWT compared with traditional treatments for trigger digit}

Of the currently accepted conservative treatments, including pain medications, orthoses, physiotherapy, and corticosteroid injection, corticosteroid injection is the most effective treatment. However, corticosteroid injection is effective in only $57 \%$ of the patients. ${ }^{6}$ Moreover, symptom recurrence at 1 year after injection is reported to be $33 \%-56 \%,{ }^{14,44,45}$ and thus, surgery is often still required. The success rate is further decreased in certain patient groups, such as diabetics. $3,44,46$ The present study, with a recurrence rate of only $6.1 \%$ at 1 -year posttreatment, would indicate a better outcome with rESWT than corticosteroid injection and all other conservative methods.

Surgery is widely accepted as the definitive treatment for trigger digit, with remission of symptoms reported to be achieved in $97 \%-100 \%$ of cases. ${ }^{14-17}$ Consequently, in the present study, rESWT was offered only to the patients with Quinnell's ${ }^{34}$ Grade 2 or 3 trigger digit, and patients with Grade 4 were referred for surgery. Therefore, only the effectiveness of rESWT in the treatment of Grade 2 and 3 conditions was commented in the present study. For these cases of trigger digit, which are not yet a fixed deformity, the recurrence rate of only $6.1 \%$ at 1 -year post-rESWT was reported. This suggests that rESWT is a promising, noninvasive alternative for the treatment of trigger digits before they become a fixed deformity (locked digit). rESWT is a conservative treatment modality without the longer recovery time and risk of complications such as nerve damage, infections, and painful scarring that are associated with surgery. ${ }^{16,17}$ Often quoted, minor complications of rESWT include bruising and redness. However, in the present study, no complications were reported, perhaps because a high pressure and high total energy flux density were not used.

Notably, in the present study, pain reductions continuously increased over the entire 1-year follow-up period. At 1-month posttreatment, the mean VAS score had reduced by $67 \%$ from baseline and at 12 months, it had reduced by $91 \%$, providing excellent long-term efficacy of rESWT. Previous studies investigating shockwave therapy for various other tendinopathies have likewise demonstrated an increasing improvement in symptoms over the entire 1-year follow-up. ${ }^{22,27}$

\section{Treatment protocol}

Although multiple, high-quality studies have demonstrated that rESWT is safe and effective in the treatment of various tendinopathies, ${ }^{19,20,22,27-31}$ controversy exists over the optimum treatment protocol that should be used in terms of shockwave generation (focused or radial), energy density, the number and frequency of treatments, method of localization (using anatomical landmarks, clinical focusing, or image-guided focusing), and the use of concomitant anaesthesia. ${ }^{27,29}$

In the present study, radial shockwaves were used because they allow the treatment of a larger tissue volume. . $1,26,27,30,47$ The pathologically changed painful area of tendon is relatively large in trigger digit compared to insertional 
tendinopathies. Thus, radial waves are advantageous as the pathologic tissue is included in the wave propagation area. Moreover, ultrasound or fluoroscopy guidance is not required with rESWT, making the application easier. ${ }^{19,26,48}$ rESWT is also less painful than focused ESWT. This makes local anesthetic unnecessary and potentially improves treatment outcome as concomitant local anesthesia has been demonstrated to reduce ESWT efficacy. ${ }^{27,28,35,36}$

With regard to pressure and the number of sessions prescribed, the protocol used in the present study was individualized to each patient. It has been demonstrated that when rESWT is used for the treatment of tendinopathy, there must be a balance between pressure and the number of sessions required. The higher the pressure, the greater the treatment effects; thus, less sessions are required, but pain during the treatment increases. The lower the pressure, the more sessions are required to have a therapeutic effect. ${ }^{49}$ Therefore, in the present study, pressure was set between 1.0 and 1.7 bars depending on each patient's individual pain tolerance. The pressure was kept low enough to ensure that the pain was tolerable, but high enough to have a therapeutic effect.

The total number of sessions prescribed was determined when symptoms resolved and ranged from 3 to 8, depending on the patient's individual response to treatment. Currently, there are no standardized guidelines for the number of rESWT sessions required when treating soft tissue conditions, ${ }^{50}$ although studies have suggested that multiple applications provide superior long-term results compared with a single application. ${ }^{51}$ Compared to other tendinopathies treated with rESWT, the finger is a rather painful area to treat. This might explain why comparatively low pressures have to be used.

\section{Strengths of the study}

This is the first retrospective cohort study to evaluate the effectiveness of rESWT for the treatment of stenosing tenosynovitis of the digital flexor tendon (trigger digit). All patients completed the 1-year follow-up. Individualization of the protocol might be a further strength and provides a possible explanation for the high efficacy of rESWT demonstrated in the present study compared to that reported by most previous studies on rESWT for tendinopathy. To the authors' knowledge, all previous studies have applied a standardized number of treatments to the patients.

\section{Limitations of the study}

Patients with a prior symptom duration ranging from 1 to 24 months were included, whereas most previous studies on rESWT have only investigated its effectiveness for more chronic tendinopathies (such as those with a prior symptomatic period of at least 6 months). ${ }^{20,21,27}$ As this study is a retrospective cohort study, it also has some inherent limitations that require consideration. There was no randomization, and there was no control group in this investigation. Furthermore, the number of subjects was relatively small in order to provide external validity to the present study. A comparative study with a control series will be more demonstrative.

\section{Recommendations for the treatment of trigger digit with rESWT}

The results demonstrate that there is no standard pressure or number of sessions that is suitable for everyone: some patients tolerate a higher pressure, and some require more treatments until recovery. With the individualized protocol, significant pain reductions were found and all patients found improvement. It was also found that pretreatment symptom duration is one of other possible factors that influence the number of sessions required by a patient. Shorter-term cases required fewer sessions and also had a lower VAS score at 1-year follow-up. Thus, the patients presenting with a longer history of symptoms need more rESWT sessions. According to the present study, varying the protocol according to each patient's tolerance and response to treatment is the best way to treat tendinopathies with rESWT.

\section{Conclusion}

The present study provides initial evidence for the effectiveness of rESWT for the treatment of trigger digit. Randomized controlled trials are required to prove well the evidence of the rESWT effect.

\section{Acknowledgments}

The authors are grateful to Storz Medical, Lohstampfestrasse 8, 8274 Tägerwilen, Switzerland, for funding the open access publication article-processing charge. The abstract of this paper was presented at the 4th Annual Podiatric Sports Medicine Conference in Association with European College of Sports \& Exercise Physicians, June 19-20, 2015, in London, UK. This paper received no specific grant from any funding agency in the public, commercial, or not-for-profit sectors. The authors affirm that this manuscript is an honest, accurate, and transparent account of the study being reported, that no important aspects of the study have been omitted, and that any discrepancies from the study as planned (and, if relevant, registered) have been explained. 


\section{Author contributions}

Malliaropoulos $\mathrm{N}$ planned the study, performed the clinical work, and contributed to writing the manuscript. Jury R collected data, performed the literature review, and contributed to writing and editing the manuscript. Pyne D, Padhiar N, and Turner J contributed to writing the manuscript. Korakakis V and Lohrer $\mathrm{H}$ contributed to writing and editing the manuscript. Meke $\mathrm{M}$ conducted the statistical analysis and contributed to writing and editing the manuscript. All authors contributed toward data analysis, drafting and critically revising the paper and agree to be accountable for all aspects of the work.

\section{Disclosure}

Lohrer $\mathrm{H}$ received research support and was a paid speaker from Storz Medical. The other authors report no conflicts of interest in this work.

\section{References}

1. Moore JS. Flexor tendon entrapment of the digits (trigger finger and trigger thumb). J Occup Environ Med. 2000;42(5):526-545.

2. Farnebo S, Chang J. Practical management of tendon disorders in the hand. Plast Reconstr Surg. 2013;132(5):841e-853e.

3. Ryzewicz M, Wolf JM. Trigger digits: principles, management, and complications. J Hand Surg Am. 2006;31(1):135-146.

4. Blyth MJ, Ross DJ. Diabetes and trigger finger. J Hand Surg Br. 1996;21(2):244-245.

5. Laszlo G, Jozsa PK. Human Tendons: Anatomy, Physiology and Pathology. Champaign (IL): Human Kinetics Publishers; 1997.

6. Fleisch SB, Spindler KP, Lee DH. Corticosteroid injections in the treatment of trigger finger: a level I and II systematic review. J Am Acad Orthop Surg. 2007;15(3):166-171.

7. Gorsche R, Wiley JP, Renger R, Brant R, Gemer TY, Sasyniuk TM. Prevalence and incidence of stenosing flexor tenosynovitis (trigger finger) in a meat-packing plant. J Occup Environ Med. 1998;40(6):556-560.

8. Trezies AJ, Lyons AR, Fielding K, Davis TR. Is occupation an aetiological factor in the development of trigger finger? J Hand Surg Br. 1998;23(4):539-540.

9. Chammas M, Bousquet P, Renard E, Poirier JL, Jaffiol C, Allieu Y. Dupuytren's disease, carpal tunnel syndrome, trigger finger, and diabetes mellitus. J Hand Surg Am. 1995;20(1):109-114.

10. Chambers RG Jr. Corticosteroid injections for trigger finger. Am Fam Physician. 2009;80(5):454.

11. Salim N, Abdullah S, Sapuan J, Haflah NH. Outcome of corticosteroid injection versus physiotherapy in the treatment of mild trigger fingers. J Hand Surg Eur Vol. 2012;37(1):27-34.

12. Benson LS, Ptaszek AJ. Injection versus surgery in the treatment of trigger finger. J Hand Surg Am. 1997;22(1):138-144.

13. Kerrigan CL, Stanwix MG. Using evidence to minimize the cost of trigger finger care. J Hand Surg Am. 2009;34(6):997-1005.

14. Wang J, Zhao JG, Liang CC. Percutaneous release, open surgery, or corticosteroid injection, which is the best treatment method for trigger digits? Clin Orthop Relat Res. 2013;471(6):1879-1886.

15. Gilberts EC, Beekman WH, Stevens HJ, Wereldsma JC. Prospective randomized trial of open versus percutaneous surgery for trigger digits. J Hand Surg Am. 2001;26(3):497-500.

16. Sato ES, Gomes Dos Santos JB, Belloti JC, Albertoni WM, Faloppa F. Treatment of trigger finger: randomized clinical trial comparing the methods of corticosteroid injection, percutaneous release and open surgery. Rheumatology. 2012;51(1):93-99.

17. Turowski GA, Zdankiewicz PD, Thomson JG. The results of surgical treatment of trigger finger. J Hand Surg Am. 1997;22(1):145-149.
18. National Guideline C. Hand, wrist, and forearm disorders, not including carpal tunnel syndrome. Available from: http://www.guideline.gov/ content.aspx?id=34435. Accessed March 23, 2015.

19. Cacchio A, Paoloni M, Barile A, et al. Effectiveness of radial shock-wave therapy for calcific tendinitis of the shoulder: single-blind, randomized clinical study. Phys Ther. 2006;86(5):672-682.

20. Rompe JD, Cacchio A, Furia JP, Maffulli N. Low-energy extracorporeal shock wave therapy as a treatment for medial tibial stress syndrome. Am J Sports Med. 2010;38(1):125-132.

21. Lohrer H, Scholl J, Arentz S. Achillodynie und Patellaspitzensyndrom - Ergebnisse der Behandlung austherapierter, chronischer Fälle mit radialen Stoßwellen. [Achilles tendinopathy and patellar tendinopathy. Results of radial shockwave therapy in patients with unsuccessfully treated tendinoses]. Sportverletz Sportschaden. 2002;16(3):108-114. German.

22. Pettrone FA, McCall BR. Extracorporeal shock wave therapy without local anesthesia for chronic lateral epicondylitis. J Bone Joint Surg Am. 2005;87(6):1297-1304.

23. Thiel M. Application of shock waves in medicine. Clin Orthop Relat Res. 2001;(387):18-21.

24. Rompe JD, Kirkpatrick CJ, Kullmer K, Schwitalle M, Krischek O. Doserelated effects of shock waves on rabbit tendo Achillis. A sonographic and histological study. J Bone Joint Surg Br. 1998;80(3):546-552.

25. Engebretsen K, Grotle M, Bautz-Holter E, Ekeberg OM, Juel NG, Brox JI. Supervised exercises compared with radial extracorporeal shock-wave therapy for subacromial shoulder pain: 1-year results of a single-blind randomized controlled trial. Phys Ther. 2011;91(1):37-47.

26. Gerdesmeyer L, Gollwitzer H, Diehl P, Wagner K. Radial extracorporeal shockwave therapy (rESWT) in orthopaedics. J Miner Stoffwechs. 2004;11:36-39.

27. Gerdesmeyer L, Frey C, Vester J, et al. Radial extracorporeal shock wave therapy is safe and effective in the treatment of chronic recalcitrant plantar fasciitis: results of a confirmatory randomized placebo-controlled multicenter study. Am J Sports Med. 2008;36(11):2100-2109.

28. Lohrer H, Nauck T, Dorn-Lange NV, Scholl J, Vester JC. Comparison of radial versus focused extracorporeal shock waves in plantar fasciitis using functional measures. Foot Ankle Int. 2010;31(1):1-9.

29. van Leeuwen MT, Zwerver J, van den Akker-Scheek I. Extracorporeal shockwave therapy for patellar tendinopathy: a review of the literature. Br J Sports Med. 2009;43(3):163-168.

30. Magosch P, Lichtenberg S, Habermeyer P. Radiale Stoßwellentherapie der Tendinosis calcarea der Rotatorenmanschette - Eine prospektive Studie. [Radial shock wave therapy in calcifying tendinitis of the rotator cuff-a prospective study]. Z Orthop Ihre Grenzgeb. 2003;141(6):629636. German.

31. Avancini-Dobrovic V, Frlan-Vrgoc L, Stamenkovic D, Pavlovic I, Vrbanic TS. Radial extracorporeal shock wave therapy in the treatment of shoulder calcific tendinitis. Coll Antropol. 2011;35(Suppl 2): 221-225.

32. Cacchio A, Rompe JD, Furia JP, Susi P, Santilli V, De Paulis F. Shockwave therapy for the treatment of chronic proximal hamstring tendinopathy in professional athletes. Am J Sports Med. 2011;39(1):146-153.

33. Moen MH, Rayer S, Schipper M, Schmikli S, Weir A, Tol JL, Backx FJ. Shockwave treatment for medial tibial stress syndrome in athletes; a prospective controlled study. Br J Sports Med. 2012;46(4):253-257.

34. Quinnell RC. Conservative management of trigger finger. Practitioner. 1980;224(1340):187-190.

35. Rompe JD, Meurer A, Nafe B, Hofmann A, Gerdesmeyer L. Repetitive low-energy shock wave application without local anesthesia is more efficient than repetitive low-energy shock wave application with local anesthesia in the treatment of chronic plantar fasciitis. J Orthop Res. 2005;23(4):931-941.

36. Labek G, Auersperg V, Ziernhold M, Poulios N, Bohler N. Einfluss von Lokalanästhesie und Energieflussdichte bei niederenergetischer Extrakorporaler Stoßwellentherapie der chronischen Plantaren Fasziitis. [Influence of local anesthesia and energy level on the clinical outcome of extracorporeal shock wave-treatment of chronic plantar fasciitis]. Z Orthop Ihre Grenzgeb. 2005;143(2):240-246. German. 
37. Roles NC, Maudsley RH. Radial tunnel syndrome: resistant tennis elbow as a nerve entrapment. J Bone Joint Surg Br. 1972;54(3):499-508.

38. Cohen J. Statistical Power Analysis for the Behavioral Sciences. 2nd ed. New Jersey: Lawrence Erlbaum Associates; 1988.

39. Faul F, Erdfelder E, Lang AG, Buchner A. G*Power 3: a flexible statistical power analysis program for the social, behavioral, and biomedical sciences. Behav Res Methods. 2007;39(2):175-191.

40. Harniman E, Carette S, Kennedy C, Beaton D. Extracorporeal shock wave therapy for calcific and noncalcific tendonitis of the rotator cuff: a systematic review. $J$ Hand Ther. 2004;17(2):132-151.

41. Gerdesmeyer L, Wagenpfeil S, Haake M, et al. Extracorporeal shock wave therapy for the treatment of chronic calcifying tendonitis of the rotator cuff: a randomized controlled trial. Jama. 2003;290(19):2573-2580.

42. Waugh CM, Morrissey D, Jones E, Riley GP, Langberg H, Screen HR. In vivo biological response to extracorporeal shockwave therapy in human tendinopathy. Eur Cell Mater. 2015;29:268-280; discussion 280.

43. Visco V, Vulpiani MC, Torrisi MR, Ferretti A, Pavan A, Vetrano M. Experimental studies on the biological effects of extracorporeal shock wave therapy on tendon models. A review of the literature. Muscles Ligaments Tendons J. 2014;4(3):357-361.

44. Rozental TD, Zurakowski D, Blazar PE. Trigger finger: prognostic indicators of recurrence following corticosteroid injection. J Bone Joint Surg Am. 2008;90(8):1665-1672.
45. Anderson B, Kaye S. Treatment of flexor tenosynovitis of the hand ('trigger finge') with corticosteroids. A prospective study of the response to local injection. Arch Intern Med. 1991;151(1):153-156.

46. Baumgarten KM, Gerlach D, Boyer MI. Corticosteroid injection in diabetic patients with trigger finger. A prospective, randomized, controlled double-blinded study. J Bone Joint Surg Am. 2007;89(12): 2604-2611.

47. Gerdesmeyer L, Maier M, Haake M, Schmitz C. Physikalisch-technische Grundlagen der extrakorporalen Stoßwellentherapie (ESWT). [Physicaltechnical principles of extracorporeal shockwave therapy (ESWT)]. Orthopade. 2002;31(7):610-617. German.

48. Kolk A, Yang KG, Tamminga R, van der Hoeven H. Radial extracorporeal shock-wave therapy in patients with chronic rotator cuff tendinitis: a prospective randomised double-blind placebo-controlled multicentre trial. Bone Joint J. 2013;95-B(11):1521-1526.

49. Lee SJ, Kang JH, Kim JY, Kim JH, Yoon SR, Jung KI. Dose-related effect of extracorporeal shock wave therapy for plantar fasciitis. Ann Rehabil Med. 2013;37(3):379-388.

50. Speed CA. Extracorporeal shock-wave therapy in the management of chronic soft-tissue conditions. J Bone Joint Surg Br. 2004;86(2):165-171.

51. Takahashi N, Ohtori S, Saisu T, Moriya H, Wada Y. Second application of low-energy shock waves has a cumulative effect on free nerve endings. Clin Orthop Relat Res. 2006;443:315-319.
Open Access Journal of Sports Medicine

\section{Publish your work in this journal}

The Open Access Journal of Sports Medicine is an international, peer-reviewed, open access journal publishing original research, reports, reviews and commentaries on all areas of sports medicine. The journal is included on PubMed. The manuscript management system is completely online and includes a very quick and fair

\section{Dovepress}

peer-review system. Visit http://www.dovepress.com/testimonials.php to read real quotes from published authors. 